\title{
Effect of care environment on educational attainment among orphaned and separated children and adolescents in Western Kenya
}

\author{
Dorothy Apedaile ${ }^{1}$, Allison DeLong², Edwin Sang ${ }^{3}$, David Ayuku4, Lukoye Atwoli ${ }^{4}, 5$, Omar Galárraga ${ }^{6}$ and \\ Paula Braitstein ${ }^{1,3^{*}}$
}

\begin{abstract}
Background: There are approximately 140 million orphaned and separated children (OSCA) around the world. In Kenya, many of these children live with extended family while others live in institutions. Despite evidence that orphans are less likely to be enrolled in school than non-orphans, there is little evidence regarding the role of care environment. This evidence is vital for designing programs and policies that promote access to education for orphans, which is not only their human right but also an important social determinant of health. The purpose of this study was to compare educational attainment among OSCA living in Charitable Children's Institutions and family-based settings in Uasin Gishu County, Kenya.
\end{abstract}

Methods: This study analyses follow up data from a cohort of OSCA living in 300 randomly selected households and 17 institutions. We used Poisson regression to estimate the effect of care environment on primary school completion among participants age $\geq 14$ as well as full and partial secondary school completion among participants age $\geq 18$. Risk ratios and 95\% confidence intervals were estimated using a bootstrap method with 1000 replications.

Results: The analysis included 1406 participants (495 from institutions, 911 from family-based settings). At baseline, $50 \%$ were female, the average age was 9.5 years, $54 \%$ were double orphans, and $3 \%$ were HIV-positive. At followup, $76 \%$ of participants age $\geq 14$ had completed primary school and $32 \%$ of participants age $\geq 18$ had completed secondary school. Children living in institutions were significantly more likely to complete primary school (aRR: 1.18, 95\% Cl: 1.10-1.28) and at least 1 year of secondary school (aRR: 1.28, 95\% Cl: 1.18-1.39) than children in family-based settings. Children living in institutions were less likely to have completed all 4 years secondary school (aRR: 0.79, 95\% Cl: 0.43-1.18) than children in family-based settings.

Conclusion: Children living in institutional environments were more likely to complete primary school and some secondary school than children living in family-based care. Further support is needed for all orphans to improve primary and secondary school completion. Policies that require orphans to leave institution environments upon their eighteenth birthday may be preventing these youth from completing secondary school.

Keywords: Education, Orphans, Children's rights, Kenya, Residential care, Institutions, Foster care, Family-based care

*Correspondence: paula.braitstein@utoronto.ca

1 Division of Epidemiology, Dalla Lana School of Public Health, University

of Toronto, 155 College Street, Toronto, ON M5T 3M7, Canada

Full list of author information is available at the end of the article

\section{Introduction}

Education is widely recognized as a fundamental social determinant of health [1-3]. Education is also recognized as a human right under Article 28 of the UN Convention on the Rights of the Child, which states that primary 
education should be free and compulsory and that secondary education should be available and accessible to all children [4]. Increased educational attainment is associated with positive health outcomes, higher socioeconomic status, greater access to healthcare, increased life expectancy, and improvements in childhood mortality [5-10].

Education has been identified as a global priority in both the Millennium Development Goals and the Sustainable Development Goals [11]. However, there continue to be disparities in educational attainment both between countries and within specific population groups. While net primary school enrolment around the world increased to $91 \%$ in 2015 from 83\% in 2000, children in the poorest households were four times less likely to attend school than children in the richest households in developing countries [12]. Sub-Saharan Africa saw the greatest increases in primary school enrolment during the Millennium Development Goals era, but continued to have the lowest literacy rates among youth compared to other regions [12].

Orphaned and separated children and youth (OSCA) have particularly low educational attainment $[13,14]$. UNICEF defines an orphan as a child under 18 years of age who has lost one or both parents to any cause of death [15]. Based on UNICEF's definition, there were an estimated 140 million orphans in the world in 2015, of whom 15.1 million had lost both parents [15]. Orphans are less likely to be attending school; more likely to have lower academic achievement and higher absenteeism; and are less likely to be in the right grade level for their age than non-orphans [16-18]. Previous research has found that there are many barriers to educational attainment for orphans, including the psychological trauma (i.e. grief and depression) and material impact (i.e. lack of money for school fees) of losing a parent [17], missing time from school to care for a sick parent before they die [17], food insecurity (i.e. a child may go to the street in search of food rather than going hungry all day at school) $[14,19]$, and the cost of school fees and ancillary costs (e.g. uniform, school shoes, school bag) [14]. Among OSCA who did not complete their educations, leaving school early has been identified as a source of significant psychological distress [19].

According to UNICEF, 52 million of the world's orphans are living in Africa [15]. Globally, an estimated 16.6 million children have lost both parents to HIV/ AIDS, of whom $90 \%$ live in sub-Saharan Africa [20]. In 2012, there were an estimated 1.8 million orphans living in Kenya, of whom $15 \%$ were double orphans who had lost both their parents [21]. The majority of OSCA live with their surviving parent or extended family [15]. However, households caring for OSCA in Kenya are often extremely poor and many are unable to meet the basic needs of the children in their care [13]. The large number of children in need of care has resulted in many children living in institutional settings (e.g., orphanages), while research from a range of countries has demonstrated negative short and long-term physical and mental health outcomes for children growing up in institutional care environments [22, 23].

However, little is known about the impact of different care environments on educational attainment, despite considerable attention to both the lack of education among OSCA as well as the negative impacts of institutions [24]. Understanding the impact of institutions and other models of care on educational attainment is vital to designing programs and policies that promote access to education for OSCA. The positive, long-term health and social impacts of increased education are particularly important OSCA, who already face discrimination and less family support $[16,25]$. The objective of this analysis was to investigate the impact of care environment on primary and secondary school completion among OSCA living in western Kenya, a country that has affirmed the right to education and the right of children to free and compulsory basic education in the constitution [26]. Due to the negative impacts of institutions in other areas of health and wellbeing, our hypothesis was that children living in institutions would complete less education than children living in family-based settings.

\section{Methods \\ Study design}

The Orphaned and Separated Children's Assessments Related to their (OSCAR's) Health and Well-Being Project is a two-phase longitudinal cohort study investigating the effects of care environment on the physical and psychosocial well-being of OSCA in Uasin Gishu County, Kenya [27]. The study enrolled participants $<18$ years of age from May 31, 2010 to April 24, 2013. Phase 1 ran from 2010 to 2015 and Phase 2 ran from 2016 to 2019. The OSCAR cohort comprises participants from 300 randomly selected households caring for OSCA and 19 Charitable Children's Institutions (CCIs) (of 21 in the county at the time of study start-up). All children, both orphaned and non-orphaned, from each household or institution were eligible to participate in the study. Among the 300 households, recruitment was designed to include 100 households receiving the government cash transfer for the support of orphaned children, 100 households not receiving the cash transfer from within the same sub-Locations as those receiving the cash transfers, and 100 households not receiving the cash transfer from different sub-Locations than those receiving the cash transfers. Sub-Locations are administrative boundaries 
within Uasin Gishu County, each headed by an Assistant Chief [27]. In-depth details about the OSCAR cohort's study design, setting, and recruitment have been previously reported [27].

\section{Study population}

This report includes all participants who were orphaned or separated at the time of enrolment into Phase 1. Separated children were defined as those whose biological mother or father was potentially alive, but functionally not part of the child's life. Since questions regarding education were introduced in Phase 2, the sample is further restricted to participants who completed at least one Phase 2 visit. Of the 19 CCIs recruiting in Phase 1, two were not eligible to participate in Phase 2 since they provided shorter-term care. Therefore, participants from these two CCIs did not enrol in Phase 2 of the OSCAR study and were not included in this analysis.

\section{Human subjects' protections}

The Moi University College of Health Sciences and Moi Teaching and Referral Hospital Institutional Research and Ethics Committee, the Indiana University Institutional Review Board, and the University of Toronto Research Ethics Boards approved this study. This study conforms to the principles embodied in the Declaration of Helsinki. Written informed consent for participation was provided by the head of household or Director of the CCI. Individual written informed assent was provided by each child aged 7 years and above. Fingerprints were used for both children and guardians who were unable to sign or write their name.

\section{Patient and public involvement in research}

This study utilized community-based, participatory processes to inform the research questions, hypotheses, and methods, as detailed elsewhere [27]. To summarize briefly, the Children's Officers in the region and representatives from CCIs were initially consulted prior to the funding application. They were requested to provide input as to whether such a study would be important from their perspective, and what their priority questions and concerns were. In addition, traditional community assemblies were held in some of the target communities to identify community concerns and priorities with respect to the care of orphaned and vulnerable children. These assemblies were also held following the initiation of the study to maintain regular contact with the community and disseminate findings. We formed an Advisory Board early on, consisting of representatives from communities, CCIs, and Children's Officers, and this board met regularly throughout the life of the study. Our study disseminated findings through the monthly Uasin Gishu
Children's Services Forum, through additional traditional community assemblies, and through the study website [28].

\section{Procedures}

Data collection was conducted in situ at CCIs and at the OSCAR Project clinic for participants from households. Annually, participants completed a standardized clinical encounter and those $\geq 10$ years of age also completed a psychosocial encounter. The clinical encounter was an enhanced well-child 'check-up' administered by the project medical officer (i.e., physician) that included a complete physical history and review of health symptoms. A psychosocial encounter measured education and employment, material well-being, behaviours and risks, peer and family relationships, and mental health. The psychosocial assessment was self-administered for those who could read and write or psychologist-administered for those that could not adequately read or write. A clinical psychologist was always available during the assessments to assist in case of questions, lack of understanding, or distress. Follow-up of cases requiring individual counselling or case management took place on a case by case basis as needed, by study staff. Household level data, including age and education level of the household head, and other characteristics of the care environments (such as shelter type and source of water) were obtained through annual site assessments administered by the Project Manager for CCIs, and Community Health Workers in the participating households.

\section{Independent variables}

The primary exposure of interest was care environment (institutional or family-based) upon study enrolment [13]. Sociodemographic characteristics were ascertained at the baseline clinical encounter, including age, sex, orphan/separated status (maternal, paternal, or both), HIV status (positive, negative, unknown), hospitalizations in the past year, area of residence (rural or urban) and time living with caregiver ( $<6$ months, 6 months- 2 years, $2-5$ years, $>5$ years, all the child's life). The guardian's level of education at enrolment (none, primary, secondary, vocational, university) was assessed through a site assessment. Follow-up time was defined as the time between the first and last encounters each individual participated in.

\section{Educational outcomes}

The Kenyan education system includes 8 years of primary school from ages 6 to 13 (Class 1 to Class 8) and 4 years of secondary school from age 14 to 17 (Form 1 to Form 4). Participants were asked to identify the highest class they had completed in school, if they had ever 
attended school, if they were currently attending school, and how many days of school they had missed in the past 4 weeks (none, $1-2$ days, $3-5$ days, $>5$ days). The primary outcomes were completion of primary school (Class 8 or higher among participants age 14 or older), completion of one or more years of secondary school (Form 1 or higher among participants age 18 or older), and completion of all 4 years of secondary school (Form 1 or higher among participants age 18 or older) at the time of the participant's last follow up visit.

\section{Statistical analysis}

Descriptive statistics at baseline were calculated for both the initial study population and the population with at least one Phase 2 visit, overall and by care environment. Mean values and standard deviations are reported for normally distributed continuous characteristics, median values and interquartile ranges are reported for nonnormally distributed continuous characteristics, and frequencies and percentages are reported for categorical characteristics. Differences in baseline characteristics by care environment were assessed using Pearson's ChiSquared tests for categorical characteristics and twosample t-tests for continuous characteristics. To assess loss to follow up, Pearson's Chi-Squared tests were used to compare categorical characteristics of participants who completed a Phase 2 visit to participants who did not complete a Phase 2 visit. Continuous characteristics were compared using a two-sample t-test. Educational outcomes at the last follow-up visit were described by frequency and percentage for each care environment.

The effect of care environment on each educational outcome (primary school completion, partial secondary school completion, and secondary school completion) was estimated separately using bootstrapped Poisson regression. Poisson regression was chosen to present a risk ratio, the ratio of the cumulative incidence of school completion in the exposed (children from CCIs) and unexposed (children from FBS) groups. Results are reported unadjusted and adjusted for sex, orphan status at enrolment, HIV status at enrolment, and hospitalization in the past year. A sensitivity analysis was conducted to adjust for area (urban or rural).

The risk ratios and 95\% confidence intervals were estimated using bootstrap resampling with 1000 replications. Sampling of participants with replacement was conducted within each original sampling stratum (CCI, non-cash transfer household, same sub-Location household, and different sub-Location household) to account for clustering. The regression models were fit using inverse probability-of-censoring weights to reduce selection bias from the differential loss to follow by simulating a pseudo-population where the loss to follow up was random [29]. These weights estimate the probability of each participant completing a Phase 2 visit based on their characteristics. The weights were calculated using generalized additive models (GAM) stratified by CCI, non-cash transfer household, same sub-Location household, and different sub-Location household. The GAMs predicted the probability of a participant completing a Phase 2 visit using a smoothed function on age at enrolment and adjusted for sex, area (urban or rural), orphan status at baseline, time with guardian at baseline, recent hospitalization at baseline, and HIV status at baseline.

\section{Results}

\section{Characteristics of participants}

Table 1 describes the baseline characteristics of the 2099 participants enrolled in the OSCAR study who were eligible to participate in Phase 2 (i.e. excluding participants from the $2 \mathrm{CCIs}$ that were not invited to participate in Phase 2). At baseline, $45 \%(n=939)$ of participants were living in a CCI while $55 \%(n=1160)$ of participants were living in a family-based setting (FBS). Among the participants living in a FBS, $36 \%(n=738)$ were living in households that received the government cash transfer (data not shown). The mean age was 10.3 years, $49 \%$ of participants were female, and there were 65 participants (3\%) who were HIV-positive at baseline.

Among participants who completed at least one Phase 2 visit, the mean age of participants from CCIs at their last Phase 2 visit was 15.5 years, while participants from FBS had a mean age of 17.3 years at their last Phase 2 visit (Table 2). Among OSCA in FBS, the majority (65\%) were living in rural areas, while $48 \%$ of OSCA in a CCI were living in rural areas. At baseline, 762 (54\%) participants were double orphans, with most double orphans living in a CCI. In FBS, 76\% of OSCA had lived with their guardian for their whole life at baseline, while $75 \%$ of OSCA from CCIs had lived with their guardian for less than 5 years at baseline. Most guardians in a CCI had vocational $(47 \%)$ or university education $(36.2 \%)$, while most guardians in a FBS had either no education (20\%) or only primary education (55\%).

Table 3 compares participants who completed a Phase 2 visit to participants who did not complete a Phase 2 visit. OSCA from FBS were significantly more likely to have completed a Phase 2 visit than OSCA from CCIs $(P<0.001)$. Participants who were younger when they enrolled in the study, living in rural areas, had at least one parent, and had lived with their guardians for longer were more likely to participate in Phase $2(P<0.001)$. HIV status at baseline and gender were not significantly associated with Phase 2 participation. 
Table 1 Population characteristics at baseline among all OSCAR participants

\begin{tabular}{|c|c|c|c|c|}
\hline & $\begin{array}{l}\text { Total } \\
N=2099 \\
N(\%)\end{array}$ & $\begin{array}{l}\mathrm{CCl} \\
N=939 \\
\mathrm{~N}(\%)\end{array}$ & $\begin{array}{l}\text { FBS } \\
N=1160 \\
N(\%)\end{array}$ & $P$-value \\
\hline $\operatorname{Age}[\operatorname{mean}(\mathrm{sd})]^{\mathrm{a}}$ & $10.3(4.7)$ & $10.2(4.65)$ & $10.3(4.74)$ & 0.99 \\
\hline Follow-up time [median (IQR)] ${ }^{\mathrm{a}}$ & $6.7(3.0-7.5)$ & $5.6(2.2-7.7)$ & $6.9(5.5-7.5)$ & $<0.001$ \\
\hline \multicolumn{5}{|l|}{ Gender } \\
\hline Female & $1035(49.3)$ & $434(46.2)$ & $601(51.8)$ & \multirow[t]{3}{*}{0.01} \\
\hline Male & $1064(50.7)$ & $505(53.8)$ & $559(48.2)$ & \\
\hline Missing & $0(0.0)$ & $0(0.0)$ & $0(0.0)$ & \\
\hline \multicolumn{5}{|l|}{ Area } \\
\hline Rural & $1145(54.5)$ & $384(40.9)$ & $761(65.6)$ & \multirow[t]{3}{*}{$<0.001$} \\
\hline Urban & $926(44.1)$ & $527(56.2)$ & $399(34.4)$ & \\
\hline Missing & $28(1.3)$ & $28(3.0)$ & $0(0.0)$ & \\
\hline \multicolumn{5}{|l|}{ Orphan Status } \\
\hline Double Orphan & $1257(59.9)$ & $811(86.4)$ & $446(38.4)$ & \multirow[t]{4}{*}{$<0.001$} \\
\hline Maternal Orphan & $187(8.9)$ & $66(7.0)$ & $121(10.4)$ & \\
\hline Paternal Orphan & $655(31.2)$ & $62(6.6)$ & $593(51.1)$ & \\
\hline Missing & $0(0.0)$ & $0(0.0)$ & $0(0.0)$ & \\
\hline \multicolumn{5}{|l|}{ Time with guardian } \\
\hline$<6$ months & $106(5.1)$ & $93(9.9)$ & $13(1.1)$ & \multirow[t]{6}{*}{$<0.001$} \\
\hline 6 months -2 years & $284(13.5)$ & $252(26.8)$ & $32(2.8)$ & \\
\hline $2-5$ years & $457(21.8)$ & $314(33.4)$ & $143(12.4)$ & \\
\hline $5+$ years & $351(16.7)$ & $234(24.9)$ & $117(10.1)$ & \\
\hline Whole life & $875(41.7)$ & $27(2.9)$ & $848(73.5)$ & \\
\hline Missing & $26(1.2)$ & $19(2.0)$ & $7(0.6)$ & \\
\hline \multicolumn{5}{|l|}{ Guardian education level } \\
\hline None & $233(11.0)$ & $0(0.0)$ & $233(20.2)$ & \multirow[t]{7}{*}{$<0.001$} \\
\hline Primary & $627(29.9)$ & $1(0.1)$ & $626(54.2)$ & \\
\hline Secondary & $375(17.9)$ & $133(14.2)$ & $242(21.0)$ & \\
\hline Vocational & $563(26.8)$ & $560(59.6)$ & $3(0.3)$ & \\
\hline University & $245(11.7)$ & $245(26.1)$ & $0(0.0)$ & \\
\hline Other & $51(2.4)$ & $0(0.0)$ & $41(4.4)$ & \\
\hline Missing & $5(0.2)$ & $0(0.0)$ & $5(0.4)$ & \\
\hline \multicolumn{5}{|l|}{ Hospitalized in past year } \\
\hline Yes & $84(4.1)$ & $55(5.9)$ & $29(2.5)$ & \multirow[t]{3}{*}{$<0.001$} \\
\hline No & 1949 (92.9) & $833(88.7)$ & $1116(97.5)$ & \\
\hline Missing & $67(3.2)$ & $51(5.4)$ & $15(1.3)$ & \\
\hline \multicolumn{5}{|l|}{ HIV Status at enrollment } \\
\hline HIV negative & $2032(96.8)$ & $895(95.3)$ & $1137(98.0)$ & \multirow[t]{3}{*}{0.001} \\
\hline HIV positive & $65(3.1)$ & $42(4.5)$ & $23(2.0)$ & \\
\hline Missing & $2(0.1)$ & $2(0.2)$ & $0(0.0)$ & \\
\hline
\end{tabular}

${ }^{\text {a }}$ reported in years

Abbreviations: CCI Charitable Children's Institution, FBS Family-Based Setting, HIV Human immunodeficiency virus

\section{Educational outcomes}

Table 4 presents educational characteristics at follow-up stratified by care environment and age group. All but 2 participants reported that they had previously attended school. Almost all participants aged 6-13 (99\%) and 14-17 (96\%) reported that they were currently attending school. Among participants over the age of 18, $94 \%$ of those from CCIs were currently attending school while $56 \%$ of those from FBS were currently attending school. Across all age groups and care environments, over $90 \%$ of participants currently attending school reported missing 2 or fewer days of school in the past 4 weeks. 
Table 2 Population characteristics among participants with at least one Phase 2 visit

\begin{tabular}{|c|c|c|c|c|}
\hline & $\begin{array}{l}\text { Total } \\
N=1406 \\
N(\%)\end{array}$ & $\begin{array}{l}\mathrm{CCl} \\
N=495 \\
N(\%)\end{array}$ & $\begin{array}{l}\text { FBS } \\
N=911 \\
N(\%)\end{array}$ & $P$-value \\
\hline Age at baseline [mean (sd)] $]^{a}$ & $9.5(4.6)$ & $8.3(4.1)$ & $10.2(4.7)$ & $<0.001$ \\
\hline Age at last visit [mean (sd)] & $16.6(4.5)$ & $15.5(3.9)$ & $17.3(4.7)$ & $<0.001$ \\
\hline Follow-up time [median (IQR)] ${ }^{\mathrm{a}}$ & $7.4(6.7-7.8)$ & $7.9(6.5-8.0)$ & $7.3(6.7-7.6)$ & $<0.001$ \\
\hline \multicolumn{5}{|l|}{ Gender } \\
\hline Female & $708(50.4)$ & $236(47.7)$ & $472(51.8)$ & \multirow[t]{3}{*}{0.14} \\
\hline Male & $698(49.6)$ & $259(52.3)$ & $439(48.2)$ & \\
\hline Missing & $0(0.0)$ & $0(0.0)$ & $0(0.0)$ & \\
\hline \multicolumn{5}{|l|}{ Area } \\
\hline Rural & $833(59.2)$ & $237(47.9)$ & $596(65.4)$ & \multirow[t]{3}{*}{$<0.001$} \\
\hline Urban & $545(38.8)$ & $230(46.5)$ & $315(34.6)$ & \\
\hline Missing & $28(2.0)$ & $28(5.7)$ & $0(0.0)$ & \\
\hline \multicolumn{5}{|l|}{ Orphan Status at baseline } \\
\hline Double Orphan & $762(54.2)$ & $434(87.7)$ & $328(36.0)$ & \multirow[t]{4}{*}{$<0.001$} \\
\hline Maternal Orphan & $131(9.3)$ & $35(7.1)$ & $96(10.5)$ & \\
\hline Paternal Orphan & $513(36.5)$ & $26(5.3)$ & $487(53.5)$ & \\
\hline Missing & $0(0.0)$ & $0(0.0)$ & $0(0.0)$ & \\
\hline \multicolumn{5}{|l|}{ Time with guardian at baseline } \\
\hline$<6$ months & $56(4.0)$ & $47(9.5)$ & $9(1.0)$ & \multirow[t]{6}{*}{$<0.001$} \\
\hline 6 months -2 years & $170(12.1)$ & $152(30.7)$ & $18(2.0)$ & \\
\hline $2-5$ years & $271(19.3)$ & $170(34.3)$ & $101(11.1)$ & \\
\hline $5+$ years & $176(12.5)$ & $92(18.6)$ & $84(9.2)$ & \\
\hline Whole life & $712(50.6)$ & $18(3.6)$ & $694(76.2)$ & \\
\hline Missing & $21(1.5)$ & $16(3.2)$ & $5(0.6)$ & \\
\hline \multicolumn{5}{|l|}{ Guardian education level } \\
\hline None & $181(12.9)$ & $0(0.0)$ & $181(19.9)$ & \multirow[t]{7}{*}{$<0.001$} \\
\hline Primary & $501(35.6)$ & $1(0.2)$ & $500(54.9)$ & \\
\hline Secondary & $268(19.1)$ & $84(17.0)$ & $184(20.2)$ & \\
\hline Vocational & $234(16.6)$ & $231(46.7)$ & $3(0.3)$ & \\
\hline University & $179(12.7)$ & $179(36.2)$ & $0(0.0)$ & \\
\hline Other & $39(2.8)$ & $0(0.0)$ & $39(4.3)$ & \\
\hline Missing & $4(0.3)$ & $0(0.0)$ & $4(0.4)$ & \\
\hline \multicolumn{5}{|l|}{ Hospitalized in past year } \\
\hline Yes & $31(2.2)$ & $10(2.0)$ & $21(2.3)$ & \multirow[t]{3}{*}{0.71} \\
\hline No & $1356(96.4)$ & $481(97.2)$ & $875(96.0)$ & \\
\hline Missing & $19(1.4)$ & $4(0.8)$ & $15(1.7)$ & \\
\hline \multicolumn{5}{|l|}{ HIV Status at enrollment } \\
\hline HIV negative & $1359(96.7)$ & $463(93.5)$ & $896(98.4)$ & \multirow[t]{3}{*}{$<0.001$} \\
\hline HIV positive & $46(3.3)$ & $31(6.3)$ & $15(1.6)$ & \\
\hline Missing & $1(0.1)$ & $1(0.2)$ & $0(0.0)$ & \\
\hline
\end{tabular}

a reported in years

Abbreviations: CCI Charitable Children's Institution, FBS Family-Based Setting, HIV Human immunodeficiency virus

For OSCA living in a CCI, 105 participants age 14-17 (63\%) and 154 participants age 18 or older $(99 \%)$ completed primary school. For OSCA living in a FBS, 118 participants age 14-17 (48\%) and 391 participants age
18 or older $(88 \%)$ completed primary school. Among participants age 18 or older from a CCI, 30 (19\%) completed all 4 years of secondary school compared to 163 (37\%) of those from FBS. However, 112 (25\%) of participants age 18 or older from FBS had not completed 
Table 3 Comparison of participants with at least one Phase 2 visit to participants that did not participate in Phase 2

\begin{tabular}{|c|c|c|c|}
\hline & $\begin{array}{l}\text { Participants without a Phase } 2 \text { Visit } \\
N=693 \\
\mathrm{~N}(\%)\end{array}$ & $\begin{array}{l}\text { Participants with a Phase } 2 \text { Visit } \\
N=1406 \\
\mathrm{~N}(\%)\end{array}$ & $p$-value \\
\hline \multicolumn{4}{|l|}{ Care environment } \\
\hline Charitable Children's Institution & $444(64.1)$ & $495(35.2)$ & \multirow[t]{2}{*}{$<0.001$} \\
\hline Family-based setting & $249(35.9 \%)$ & $911(64.8)$ & \\
\hline Age at baseline $[\text { mean }(\mathrm{sd})]^{\mathrm{a}}$ & $11.7(4.6)$ & $9.5(4.6)$ & $<0.001$ \\
\hline Age at last visit [mean (sd)] ${ }^{\mathrm{a}}$ & $13.7(4.8)$ & $16.7(4.5)$ & $<0.001$ \\
\hline \multicolumn{4}{|l|}{ Gender } \\
\hline Female & $327(47.2)$ & $708(50.4)$ & \multirow[t]{3}{*}{0.17} \\
\hline Male & $366(52.8)$ & $698(49.6)$ & \\
\hline Missing & $0(0.0)$ & $0(0.0)$ & \\
\hline \multicolumn{4}{|l|}{ Area } \\
\hline Rural & $312(45.0)$ & $833(59.2)$ & \multirow[t]{3}{*}{$<0.001$} \\
\hline Urban & $381(55.0)$ & $545(38.8)$ & \\
\hline Missing & $0(0.0)$ & $28(2.0)$ & \\
\hline \multicolumn{4}{|l|}{ Orphan status at baseline } \\
\hline Double Orphan & $495(71.4)$ & $762(54.2)$ & \multirow[t]{4}{*}{$<0.001$} \\
\hline Maternal Orphan & $56(8.1)$ & $131(9.3)$ & \\
\hline Paternal Orphan & $142(20.5)$ & $513(36.5)$ & \\
\hline Missing & $0(0.0)$ & $0(0.0)$ & \\
\hline \multicolumn{4}{|l|}{ Time with guardian at baseline } \\
\hline$<6$ months & $50(7.2)$ & $56(4.0)$ & \multirow[t]{6}{*}{$<0.001$} \\
\hline 6 months -2 years & $114(16.5)$ & $170(12.1)$ & \\
\hline $2-5$ years & $186(26.8)$ & $271(19.3)$ & \\
\hline $5+$ years & $175(25.3)$ & $176(12.5)$ & \\
\hline Whole life & $163(23.5)$ & $712(50.6)$ & \\
\hline Missing & $5(0.7)$ & $21(1.5)$ & \\
\hline \multicolumn{4}{|l|}{ Guardian education level } \\
\hline None & $52(7.5)$ & $181(12.9)$ & \multirow[t]{7}{*}{$<0.001$} \\
\hline Primary & $126(18.2)$ & $501(35.6)$ & \\
\hline Secondary & $107(15.4)$ & $268(19.1)$ & \\
\hline Vocational & $329(47.5)$ & $234(16.6)$ & \\
\hline University & $66(9.5)$ & $179(12.7)$ & \\
\hline Other & $12(1.7)$ & $39(2.8)$ & \\
\hline Missing & $1(0.1)$ & $4(0.3)$ & \\
\hline \multicolumn{4}{|l|}{ HIV Status at baseline } \\
\hline HIV negative & $673(97.1)$ & $1359(96.7)$ & \multirow[t]{3}{*}{0.51} \\
\hline HIV positive & $19(2.7)$ & $46(3.3)$ & \\
\hline Missing & $1(0.1)$ & $1(0.1)$ & \\
\hline
\end{tabular}

a reported in years

Abbreviations: HIV Human immunodeficiency virus

any secondary school compared to $8(5.1 \%)$ participants from a CCI.

The results of the Poisson regression with inverse probability weights are presented in Table 5. Area (rural/ urban) did not change the main effect and was not included in the final model. Among participants aged 14 and over, OSCA living in a CCI were 1.18 times more likely to complete primary school (95\% CI 1.10-1.28) than OSCA living in a FBS after adjusting for potential confounders. Among participants aged 18 and over, OSCA living in a CCI were also more likely to complete one or more years of secondary school compared to OSCA living in a FBS (aRR $=1.28,95 \%$ CI 1.18-1.39). Although OSCA age $18+$ in a CCI were 0.79 times as 
Table 4 Educational participation in school-aged children and youth at last follow up visit

\begin{tabular}{|c|c|c|c|c|c|c|}
\hline \multirow{2}{*}{ 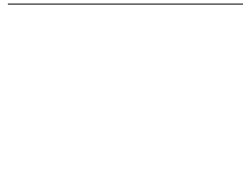 } & \multicolumn{3}{|l|}{$\mathrm{CCl}$} & \multicolumn{3}{|l|}{ FBS } \\
\hline & $\begin{array}{l}\text { Age 6-13 } \\
N=172 \\
N(\%)\end{array}$ & $\begin{array}{l}\text { Age 14-17 } \\
N=167 \\
N(\%)\end{array}$ & $\begin{array}{l}\text { Age 18+ } \\
N=156 \\
\mathrm{~N}(\%)\end{array}$ & $\begin{array}{l}\text { Age 6-13 } \\
N=220 \\
\mathrm{~N}(\%)\end{array}$ & $\begin{array}{l}\text { Age 14-17 } \\
N=244 \\
\mathrm{~N}(\%)\end{array}$ & $\begin{array}{l}\text { Age } 18+ \\
N=444 \\
\mathrm{~N}(\%)\end{array}$ \\
\hline \multicolumn{7}{|c|}{ Ever attended school } \\
\hline Yes & $172(100.0)$ & $167(100.0)$ & $156(100.0)$ & $218(99.1)$ & $244(100.0)$ & $444(100.0)$ \\
\hline Missing & $0(0.0)$ & $0(0.0)$ & $0(0.0)$ & $0(0.0)$ & $0(0.0)$ & $0(0.0)$ \\
\hline \multicolumn{7}{|c|}{ Currently attending school } \\
\hline Yes & $171(99.4)$ & $166(99.4)$ & $146(93.6)$ & $216(98.2)$ & $230(94.3)$ & $247(55.6)$ \\
\hline Missing & $0(0.0)$ & $0(0.0)$ & $0(0.0)$ & $2(0.9)$ & $0(0.0)$ & $0(0.0)$ \\
\hline \multicolumn{7}{|c|}{ Days of school missed in past 4 weeks ${ }^{a}$} \\
\hline None & $148(86.5)$ & $154(92.8)$ & $132(90.4)$ & $194(89.8)$ & $198(86.1)$ & $208(84.2)$ \\
\hline $1-2$ days & $19(11.1)$ & $10(6.0)$ & $5(3.4)$ & $14(6.5)$ & $18(7.8)$ & $14(5.7)$ \\
\hline $3-5$ days & $3(1.7)$ & $1(0.6)$ & $4(2.7)$ & $3(1.4)$ & $7(3.0)$ & $8(3.2)$ \\
\hline$>5$ days & $1(0.6)$ & $1(0.6)$ & $5(3.4)$ & $2(0.9)$ & $6(2.6)$ & $13(5.2)$ \\
\hline Missing & $0(0.0)$ & $0(0.0)$ & $0(0.0)$ & $5(2.3)$ & $1(0.4)$ & $0(0.0)$ \\
\hline \multicolumn{7}{|l|}{ Primary school } \\
\hline None completed & $15(8.7)$ & $0(0.0)$ & $0(0.0)$ & $19(8.6)$ & $2(0.8)$ & $1(0.2)$ \\
\hline Some completed ${ }^{b}$ & $153(88.9)$ & $62(37.1)$ & $2(1.3)$ & $195(88.6)$ & $123(50.4)$ & $52(11.7)$ \\
\hline All completed ${ }^{c}$ & $2(1.2)$ & $105(62.9)$ & $154(98.7)$ & $2(0.9)$ & $118(48.4)$ & $391(88.1)$ \\
\hline Missing & $2(1.2)$ & $0(0.0)$ & $0(0.0)$ & $4(1.8)$ & $1(0.4)$ & $0(0.0)$ \\
\hline \multicolumn{7}{|l|}{ Secondary school } \\
\hline None completed & NA & $97(58.1)$ & $8(5.1)$ & NA & $169(69.3)$ & $112(25.2)$ \\
\hline Some completed ${ }^{\mathrm{d}}$ & NA & $70(41.9)$ & $118(75.6)$ & NA & $71(29.1)$ & $169(38.1)$ \\
\hline All completed ${ }^{e}$ & NA & $0(0.0)$ & $30(19.2)$ & NA & $3(1.2)$ & $163(36.7)$ \\
\hline Missing & NA & $0(0.0)$ & $0(0.0)$ & NA & $1(0.4)$ & $0(0.0)$ \\
\hline
\end{tabular}

${ }^{\text {a }}$ Among participants currently attending school

${ }^{\mathrm{b}}$ Highest level completed is between Grade 1 and Grade 7

c Highest level completed is Grade 8 or higher

${ }^{\mathrm{d}}$ Highest level completed is between Form 1 and Form 3

e Highest level completed is Form 4 or higher

Abbreviations: CCI Charitable Children's Institution, FBS Family-Based Setting

Table 5 Effect of Care Environment on Educational Attainment at last follow-up visit from Poisson regression analyses

\begin{tabular}{|c|c|c|c|c|c|c|}
\hline \multirow[t]{2}{*}{ Characteristic } & \multicolumn{2}{|c|}{ Primary Completion $^{a}$} & \multicolumn{2}{|c|}{$\begin{array}{l}\text { Completed } 4 \text { Years of Secondary } \\
\text { School }^{\mathrm{b}}\end{array}$} & \multicolumn{2}{|c|}{$\begin{array}{l}\text { Completed } 1+\text { Years of } \\
\text { Secondary School }\end{array}$} \\
\hline & $\mathrm{RR}(95 \% \mathrm{Cl})$ & $\operatorname{aRR}(95 \% \mathrm{CI})^{c}$ & RR $(95 \% \mathrm{Cl})$ & $\operatorname{aRR}(95 \% \mathrm{CI})^{c}$ & RR $(95 \%$ Cl) & $\operatorname{aRR}(95 \% \mathrm{CI})^{c}$ \\
\hline Environment (CCI vs FBS) & $1.17(1.10-1.24)$ & $1.18(1.10,1.28)$ & $0.86(0.44,1.37)$ & $0.79(0.43,1.18)$ & $1.29(1.21,1.36)$ & $1.28(1.18,1.39)$ \\
\hline Sex (Male vs Female) & & $0.90(0.84,0.96)$ & & $0.65(0.46,0.96)$ & & $0.93(0.87,0.99)$ \\
\hline \multicolumn{7}{|l|}{ Orphan status } \\
\hline Double orphan (ref) & & 1.00 (ref) & & 1.00 (ref) & & 1.00 (ref) \\
\hline Maternal orphan & & $0.93(0.80,1.05)$ & & $0.85(0.50,1.28)$ & & $1.03(0.90,1.16)$ \\
\hline Paternal orphan & & $1.04(0.95,1.12)$ & & $0.92(0.69,1.23)$ & & $1.01(0.92,1.11)$ \\
\hline HIV Positive & & $0.94(0.75,1.10)$ & & $0.98(0.32,1.76)$ & & $1.03(0.82,1.20)$ \\
\hline Hospitalized in past year & & $1.03(0.84,1.20)$ & & $0.72(0.20,1.38)$ & & $0.88(0.59,1.12)$ \\
\hline
\end{tabular}

${ }^{a}$ Among participants aged 14 or older

${ }^{\mathrm{b}}$ Among participants aged 18 or older

' Adjusted for sex, orphan status, HIV status, and hospitalization in the past year

Abbreviations: RR Risk ratio, CI Confidence interval, aRR Adjusted risk ratio, CCI Charitable Children's Institution, FBS Family-Based Setting 
likely to complete all 4 years of secondary school when compared to those in a FBS (95\% CI 0.43-1.18) after adjustment, this result was not statistically significant.

\section{Discussion}

Our findings suggest that there is room for improvement in both primary and secondary school completion for OSCA in Uasin Gishu County, Kenya, regardless of care environment. While most school-aged OSCA were currently attending school, many had not achieved the educational milestones expected for their age. This was particularly pronounced in OSCA over the age of 18, of whom only a third had completed high school. OSCA living in a CCI were significantly more likely to complete primary school (among participants aged 14 and over) and at least 1 year of secondary school (among participants aged 18 and over) than those living in family-based care, after adjusting for potential confounding variables. There was no statistically significant effect of care environment on secondary school completion among participants aged 18 and over after adjustment.

Previous research has found that orphans are less likely to be in the right grade for their age than non-orphans [30]. While we did not compare orphans to non-orphans in this study, our results suggest that many OSCA are completing primary school later than expected, despite high attendance and low absenteeism. This somewhat contradicts other studies that found high absenteeism among orphans and lower school enrollment and attendance $[14,30,31]$. These differences may be explained by Kenya's system of free primary education, which was re-introduced in 2003 during the era of the Millennium Development Goals [32]. However, there continue to be concerns regarding quality, class sizes, other fees, and physical capacity in schools [32]. There also continue to be financial barriers to attending secondary school, particularly for OSCA who often live in low-income households [14, 31]. Very few OSCA in our study completed all 4 years of secondary school and one fifth had completed no secondary school at all by the time they had turned 18.

Although institutions have been associated with a wide range of negative impacts on OSCA, some research has found that health, emotional, and cognitive functioning were no worse for children living in institutions compared to those living in the community [33, 34]. Consistent with these findings, we found that OSCA living in a CCI were more likely to complete primary school and one or more years of secondary school compared to children living in family-base care. Potential explanations for this include higher education levels among guardians in institutions compared to guardians in FBS and fewer financial barriers to attending school due to fees being covered by the CCI [35]. In addition, OSCA living in a $\mathrm{CCI}$ may have more time for school due to fewer responsibilities at home and less need to earn income [13].

Despite consistently higher primary school and partial secondary school completion, OSCA from CCIs were less likely to fully complete secondary school, though this result was not statistically significant. It is possible that their education is being interrupted due to "aging out" of care when they turn 18 and needing to find their own shelter and employment [36]. In contrast, OSCA from FBS may be able to stay in their households and continue attending school with the support of their extended family. While government policy requires that CCI's develop exit plans for youth, the transition can be difficult and youth may lack the support needed to complete their studies [25]. In contrast, youth living in a FBS are not necessarily required to move out upon turning 18 . This impact of "aging out" of care is not unique to Kenya's CCI system. Youth aging out of state foster care in Canada have low rates of academic achievement and report a sense of anxiety and abandonment [37, 38]. In the United States, one quarter of former foster youth age 23-24 did not have a high school diploma. Youth who remained in care after the age of 18 reported better educational and employment outcomes [39].

This study has several strengths. The sampling design allows us to compare educational outcomes for OSCA living in institutions to those of OSCA living in familybased settings within the same geographical region. Detailed information was collected on study participants, allowing us to adjust for many important confounding variables. This study also has some limitations. Educational outcomes were self-reported by participants and are subject to recall bias and reporting bias. We were also unable to measure education quality or achievement. In addition, follow up time was shorter for OSCA from CCIs compared to those from family-based settings due to difficulties in following youth once they aged out of care. Thus, these results reflect the participants who remained in the OSCAR study long enough to complete at least one Phase 2 visit.

In conclusion, our results demonstrate that OSCA living in institutional environments are significantly more likely to complete primary school and attend secondary school but are somewhat less likely to complete secondary school than OSCA living in family-based care. For these children and youth who already face discrimination and very little to no support from their families, education may be especially important for future success. Further action is needed to reduce barriers to secondary school and improve completion rates among all OSCA. Policies forcing youth living in CCIs to leave upon turning 18 may pose a significant barrier to secondary school 
completion. Lessons from other countries demonstrate that OSCA continue to need support after turning 18. Efforts to improve care for OSCA in Kenya must build on strengths in the systems that currently exist and avoid replicating limitations seen in other jurisdictions.

\begin{abstract}
Acknowledgements
We wish to gratefully acknowledge the Chiefs, Assistant Chiefs, and Village Elders of the Locations of Pioneer, Kapsoya, Koisagat, Ol'Lenguse, Olare, Tarakwa, Kipsinende and Kapyemit for their support and leadership. We also wish to acknowledge all the residents of these Locations and particularly the many households caring for vulnerable members of their community, notably orphaned and separated children, children with physical and mental disabilities, and the elderly. We particularly want to acknowledge the County and District Children's Officers, particularly Mr. Philip Nzenge, for their dedication to protecting the children of Uasin Gishu County, as well as the Uasin Gishu Children's Services Forum. This study would not have been possible without the willing participation of the children and youth participants and their guardians and we would like to acknowledge and thank them. The enthusiasm and commitment of the staff and volunteers of the OSCAR project have not gone unnoticed and we the authors wish to thank them for their efforts and engagement with this project.
\end{abstract}

\section{Authors' contributions}

D. Ayuku, LA, OG, and PB contributed to the conceptualization and design of the study, data acquisition, and interpretation of the results. D. Apedaile and $\mathrm{AD}$ conducted the analysis and contributed to the conceptualization of the research question. ES contributed to the data acquisition. D. Apedaile wrote the first draft of the manuscript. All authors contributed to the interpretation of the results, revised the manuscript, and approved the final manuscript for submission.

\section{Funding}

This project was supported by the Eunice Kennedy Shriver National Institute of Child Health \& Human Development [R01HD060478]. The content is solely the responsibility of the authors and does not necessarily represent the official views of the Eunice Kennedy Shriver National Institute of Child Health \& Human Development or the National Institutes of Health. The funder of the study had no role in study design, data collection, data analysis, data interpretation, or writing of the report.

\section{Availability of data and materials}

The datasets analysed during this study are not publicly available to protect individual privacy but are available from the corresponding author on reasonable request.

\section{Declarations}

\section{Ethics approval and consent to participate}

The Moi University College of Health Sciences and Moi Teaching and Referral Hospital Institutional Research and Ethics Committee, the Indiana University Institutional Review Board, and the University of Toronto Research Ethics Boards approved this study. This study conforms to the principles embodied in the Declaration of Helsinki. Written informed consent for participation was provided by the head of household or Director of the CCI. Individual written informed assent was provided by each child aged 7 years and above. Fingerprints were used for both children and guardians who were unable to sign or write their name.

\section{Consent for publication}

Not applicable.

\section{Competing interests}

The authors declare that they have no competing interests.

\section{Author details}

'Division of Epidemiology, Dalla Lana School of Public Health, University of Toronto, 155 College Street, Toronto, ON M5T 3M7, Canada. ${ }^{2}$ Department of Biostatistics, School of Public Health, Brown University, Providence, RI, USA. ${ }^{3}$ Academic Model Providing Access to Healthcare (AMPATH), Eldoret, Kenya. ${ }^{4}$ Department of Mental Health and Behavioural Sciences, College of Health Sciences, School of Medicine, Moi University, Eldoret, Kenya. ${ }^{5}$ Aga Khan University Medical College, East Africa, Nairobi, Kenya. ${ }^{6}$ Department of Health Policy, Services, and Practice, School of Public Health, Brown University, Providence, RI, USA.

Received: 30 July 2021 Accepted: 10 December 2021

Published online: 18 January 2022

\section{References}

1. Marmot M, Friel S, Bell R, Houweling TA, Taylor S. Closing the gap in a generation: health equity through action on the social determinants of health. Lancet. 2008;372(9650):1661-9.

2. Hahn RA, Truman BI. Education improves public health and promotes health equity. Int J Health Serv Plan Adm Eval. 2015;45(4):657-78.

3. Education: a neglected social determinant of health. Lancet Public Health. 2020;5(7):e361.

4. UN General Assembly. Convention on the Rights of the Child. 1989.

5. Raghupathi V, Raghupathi W. The influence of education on health: an empirical assessment of OECD countries for the period 1995-2015. Arch Public Health. 2020;78(1):20

6. Hummer RA, Hernandez EM. The effect of educational attainment on adult mortality in the United States*. Popul Bull. 2013;68(1):1-16.

7. Parker M, Bucknall M, Jagger C, Wilkie R. Population-based estimates of healthy working life expectancy in England at age 50 years: analysis of data from the English longitudinal study of ageing. Lancet Public Health. 2020:5(7):e395-403.

8. Wu Y-T, Daskalopoulou C, Terrera GM, Niubo AS, Rodríguez-Artalejo F, Ayuso-Mateos JL, et al. Education and wealth inequalities in healthy ageing in eight harmonised cohorts in the ATHLOS consortium: a population-based study. Lancet Public Health. 2020;5(7):e386-94.

9. Jeong J, Kim R, Subramanian SV. How consistent are associations between maternal and paternal education and child growth and development outcomes across 39 low-income and middle-income countries? J Epidemiol Community Health. 2018;72(5):434-41.

10. Gakidou E, Cowling K, Lozano R, Murray CJ. Increased educational attainment and its effect on child mortality in 175 countries between 1970 and 2009: a systematic analysis. Lancet. 2010;376(9745):959-74.

11. United Nations. The sustainable development goals report -2020 [internet]. 2020. Available from: https://sdgs.un.org/sites/default/files/2020-09/ The-Sustainable-Development-Goals-Report-2020.pdf

12. United Nations. The Millenium Development Goals Report 20152015. [Cited 2020 Oct 9]. Available from: https://www.un.org/millenniumgoals/ 2015_MDG_Report/pdf/MDG\%202015\%20rev\%20(July\%201).pdf

13. Embleton L, Ayuku D, Kamanda A, Atwoli L, Ayaya S, Vreeman R, et al. Models of care for orphaned and separated children and upholding children's rights: cross-sectional evidence from western Kenya. BMC Int Health Hum Rights. 2014;14(1):9.

14. Skovdal M, Evans R. The emergence of an ethic of care in rural Kenyan schools? Perspectives of teachers and orphaned and vulnerable pupils. Child Geogr. 2017;15(2):160-76.

15. UNICEF. Orphans. [Cited 2020 Oct 9]. Available from: https://www.unicef. org/media/orphans

16. Raymond JM, Zolnikov TR. AIDS-affected orphans in sub-Saharan Africa: a scoping review on outcome differences in rural and urban environments. AIDS Behav. 2018;22(10):3429-41.

17. Bicego $G$, Rutstein $S$, Johnson K. Dimensions of the emerging orphan crisis in sub-Saharan Africa. Soc Sci Med. 2003;56(6):1235-47.

18. Skovdal M, Webale A, Mwasiaji W, Tomkins A. The impact of communitybased capital cash transfers on orphan schooling in Kenya. Dev Pract. 2013;23(7):934-43.

19. Ntuli B, Mokgatle M, Madiba S. The psychosocial wellbeing of orphans: the case of early school leavers in socially depressed environment in Mpumalanga Province, South Africa. Plos One. 2020;15(2):e0229487.

20. Joint United Nations Programme on HIV/AIDS (UNAIDS). Global report: UNAIDS report on the global AIDS epidemic 2010. Geneva: UNAIDS; 2010 
21. Lee VC, Muriithi P, Gilbert-Nandra U, Kim AA, Schmitz ME, Odek J, et al. Orphans and vulnerable children in Kenya: results from a nationally representative population-based survey. J Acquir Immune Defic Syndr. 2014;66(Suppl 1):S89-97.

22. Boyce N, Godsland J, Sonuga-Barke E. Institutionalisation and deinstitutionalisation of children: the executive summary from a lancet group commission. Lancet Child Adolesc Health. 2020:4(8):562-3.

23. Berens $A E$, Nelson CA. The science of early adversity: is there a role for large institutions in the care of vulnerable children? Lancet. 2015;386(9991):388-98.

24. van IJzendoorn MH, Bakermans-Kranenburg MJ, Duschinsky R, Fox NA, Goldman PS, Gunnar MR, et al. Institutionalisation and deinstitutionalisation of children 1: a systematic and integrative review of evidence regarding effects on development. Lancet Psychiatry. 2020;7(8):703-20.

25. Gayapersad A, Ombok C, Kamanda A, Tarus C, Ayuku D, Braitstein P. The production and reproduction of kinship in charitable Children's institutions in Uasin Gishu County, Kenya. Child Youth Care Forum. 2019;48(6):797-828.

26. Republic of Kenya. Constitution of Kenya. 2010.

27. Kamanda A, Embleton L, Ayuku D, Atwoli L, Gisore P, Ayaya S, et al. Harnessing the power of the grassroots to conduct public health research in sub-Saharan Africa: a case study from western Kenya in the adaptation of community-based participatory research (CBPR) approaches. BMC Public Health. 2013;13(1):91.

28. The OSCAR Study. 2021. [Cited 2021 Nov 5]. Available from: https://oscar cohort.com/

29. Howe CJ, Cole SR, Lau B, Napravnik S, Eron JJJ. Selection Bias due to loss to follow up in cohort studies. Epidemiology. 2016;27(1):91-7.

30. Guo Y, Li X, Sherr L. The impact of HIV/AIDS on children's educational outcome: a critical review of global literature. AIDS Care. 2012;24(8):993-1012.

31. Evans DK, Edward M. Orphans and schooling in Africa: a longitudinal analysis. Demography. 2007:44(1):35-57.

32. Oketch MO, Somerset HCA. Free primary education and after in Kenya: enrolment impact, quality effects, and the transition to secondary school. Falmer: Consortium for Research on Educational Access, Transitions and Equity; 2010.

33. Whetten K, Ostermann J, Whetten RA, Pence BW, O'Donnell K, Messer LC, et al. A comparison of the wellbeing of orphans and abandoned children ages 6-12 in institutional and community-based care settings in 5 less wealthy nations. Plos One. 2009;4(12):e8169.

34. Braitstein P. Institutional Care of Children in low- and middle-income settings: challenging the conventional wisdom of Oliver twist. Glob Health Sci Pract. 2015;3(3):330-2.

35. Pufall E, Eaton JW, Nyamukapa C, Schur N, Takaruza A, Gregson S. The relationship between parental education and children's schooling in a time of economic turmoil: the case of East Zimbabwe, 2001 to 2011. Int J Educ Dev. 2016;51:125-34.

36. UNICEF, Republic of Kenya. National Standards for Best Practices in Charitable Children's Institutions. 2013 [Cited 2021 Feb 20]. Available from: https://bettercarenetwork.org/sites/default/files/National\%20Standards\% 20for\%20Best\%20Practices\%20in\%20Charitable\%20Children\%27s\%20Ins titutions.pdf

37. Kovarikova J. Exploring youth outcomes after aging-out of care. Toronto: Office of the Provincial Advocate for Children and Youth; 2017.

38. Brownell MD, Roos NP, MacWilliam L, Leclair L, Ekuma O, Fransoo R. Academic and social outcomes for high-risk youths in Manitoba. Can J Educ. 2010;33(4):804-36.

39. Hook JL, Courtney ME. Employment outcomes of former foster youth as young adults: the importance of human, personal, and social capital. Child Youth Serv Rev. 2011;33(10):1855-65.

\section{Publisher's Note}

Springer Nature remains neutral with regard to jurisdictional claims in published maps and institutional affiliations.
Ready to submit your research? Choose BMC and benefit from:

- fast, convenient online submission

- thorough peer review by experienced researchers in your field

- rapid publication on acceptance

- support for research data, including large and complex data types

- gold Open Access which fosters wider collaboration and increased citations

- maximum visibility for your research: over $100 \mathrm{M}$ website views per year

At BMC, research is always in progress.

Learn more biomedcentral.com/submissions 